\title{
Variation Analysis of Three Dimensional non-rigid Assemblies
}

\author{
Byungwoo Lee, Mohammed M. Shalaby, Ronald J. Collins, Victor Crisan, \\ Stephen A. Walls, Dean M. Robinson and Kazuhiro Saitou, Member, IEEE
}

\begin{abstract}
Variation analysis methods are, currently, more widely used during new product development to greatly reduce downstream rework and/or design changes. This is significantly important when considering large, built up sheet or thin plate "flexible" assemblies as those currently used in automotive or aerospace industries. Whereas methods to take flexibility into account can be found in literature, there are few addressing detailed process for three-dimensional assembly of industrial complexity. This paper presents a streamlined procedure for variation analysis of a complex assembly that integrates Datum Flow Chain analysis, a commercial three-dimensional variation analysis and FEA. The procedure is applied to a realistic industry case, a commercial airplane's wing-box assembly to determine the effect of part variation and flexibility on the assembly's variation. The case study shows that a structural enclosure such as the wing-box assembly is robust against pull-up forces applied during assembly operations.
\end{abstract}

\section{INTRODUCTION}

$\mathrm{A}$ $\mathrm{N}$ assembly is a set of parts sharing mating features (joints) among them. Due to each part's manufacturing variations, the mating features are neither in perfect shape nor in perfect location and orientation. As a result, mismatch (gap or interference) exists between mating features of two parts being joined. One method to minimize such mismatches is to design joints and assembly sequence such that parts constrain each other exactly. And, assemblies mainly consisting of sheet metals and beams are typically exactly constrained for in-plane or in-line degree-of-freedom (DOF), using locating features such as pin-hole or pin-slot. However, for out-of-plane DOFs, long flanges such as lap joints between a pair of sheet metals almost makes it impossible to exactly constrain each other, because it is very hard to keep the long flanges in perfect form. One can constrain those flexible parts' out-of-plane DOFs by having contact in a small area and shim the gap for the rest of the mating feature, but obviously it is not an economical option. Instead, parts are pulled up to close the mismatch and clamped, taking advantage of parts' flexibility. Although this is probably the most economical solution to put together long and slender flexible parts, pull-up itself deforms parts and the residual

Manuscript received December 15, 2006.

B. Lee and D. M. Robinson are with the Global Research Center, General Electric Company, Niskayuna, NY 12309 USA. (phone 518-387-7465; fax 518-387-5752; e-mail: leeb@research.ge.com, robinsondm@crd.ge.com).

R. J. Collins, V. Crisan and S. A. Walls are with Boeing Company, Everett, WA 98204 USA. (e-mail: ronald.j.collins@boeing.com, victor.crisan@boeing.com, stephen.a.walls@boeing.com)

M. M. Shalaby and K. Saitou are with the Mechanical Engineering Department, University of Michigan, Ann Arbor, MI 48103 USA (e-mail: mshalaby@umich.edu,kazu@umich.edu). force by the pull-up drives spring-back of the assembled structure.

In large-scale assembly manufacturing industries, commercial three-dimensional variation analysis (3DVA) softwares are being adopted to simulate variation propagation in assemblies. Although some of them include capability to incorporate finite element analysis (FEA) for flexible parts based on theories developed in academia [1]-[5], holistic process map to integrate assembly design analysis, 3DVA and FEA has not been addressed. This paper provides a detailed process on how to analyze an assembly design and sequence, and use a commercial 3DVA and FEA, through a case study of a commercial airplane's wing box assembly.

\section{RELATED WORK}

Numerous works have been done in the area of variation analysis of compliant assembly. The major assumption made is the pull-up forces are linearly dependent on potential variations, as variations are usually very small compared to nominal dimension. The early works of $\mathrm{Hu}$ and his colleagues[1]-[3] provide a starting point for many following literature on linearized method of flexible assembly. They use the method of influence coefficient to obtain stiffness matrices, for the stack-up analysis of sheet metal assemblies.

Reference [4] proposes an approach that directly computes an assembly's shape using condensed stiffness matrices (super elements). Yet, the approach assumes parts are fixed to rigid grounds. A generic computational framework that can facilitate a variation simulation of compliant assembly is proposed in [5]. Reference [6] combines the use of principal component analysis (PCA) and finite element method (FEM) in estimating the effect of part/component variation on assembly variation. The recent work by [7] explicitly discusses the independency between variation sources to reduce the modeling complexity through six theoretical rationales.

Several methods have been proposed in literature to properly constrain assemblies, including: Minimum Constraint Design [8] and Exact Constraint Design [9]-[11]. Whitney and his colleagues [12]-[14] promoted the exact (or proper) constraint design in the domain of type 2 assemblies (assemblies using wash-out joints) and developed the theory of Datum Flow Chain (DFC).

Variation propagation in a multi-station assembly system has been modeled by a number of researchers, using state space models [15]-[17]. These works consider part variation and tooling variation, they do not take compliance into account. Reference [18] incorporates the sensitivity matrix 
[2] into state space model, to include the effect of compliance.

All of the works reviewed runs upfront FEA to obtain constant and linear stiffness matrix between deformation and force and use it throughout Monte Carlo simulation instead of running FEA for each iteration. This approach reduces computational time by several orders of magnitude.

\section{METHOD}

Fig. 1 illustrates typical stages in an assembly step of flexible parts [1]-[5]. A part is placed on another part or a fixture, pull-up to close the mismatch or bring each mating feature to its nominal (clamping) location, where they are fastened. Then, the completed assembly is released from clamps and fixtures. The location of a point to be measured will be displaced during these stages. Fig 2. depicts an overall process to estimate the final location of the point. Details are described in the following subsections.

(a)

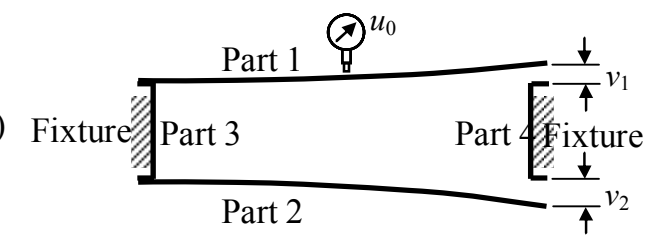

(b)

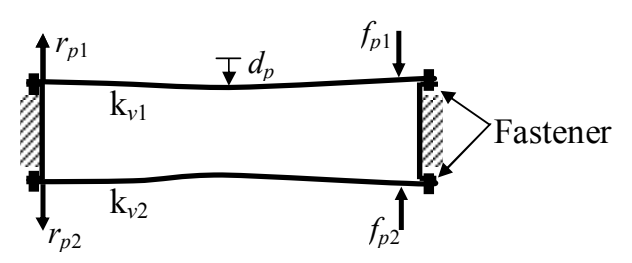

(c)

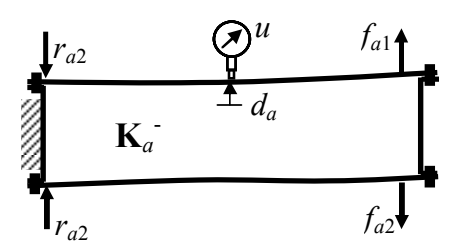

Fig. 1. Steps to estimate assembly variation with flexible parts: (a) initial position, (b) clamping deformation, and (c) spring-back deformation.

\section{A. Analyzing assembly to identify over-constraints}

The first step is to analyze the assembly sequence, locating scheme and joint geometry to identify what joint over-constrains what DOFs. In Fig. 1, it is obvious to see that part 1 and part 2 are located to part 3 and the mating features at the other end will over-constrain the part in vertical and rotational DOF when clamped. However, it may not be obvious for a complex, multi-stage assembly with fixtures, where DFC and Screw Theory can be used to identify over-constraining joints and DOFs [12]-[14], [19], [20].

\section{B. Setting-up $3 D V A$}

The next step is to measure the mismatch $(v)$ between each joint to its nominal (or clamping) position for each DOF that will be over-constrained by the joint. If a rotational DOF is over-constrained by the joint, angular mismatch should be measured. As shown in Fig. 1, if one part is much more flexible than the other in a DOF, simply the mismatch between the joint location can measured, as the flexible part will be pulled up to the rigid part. Because there are various contributing variations to mismatches for complex 3D assemblies, a 3DVA software is a good tool to measure these mismatches as the parts are stacked up as rigid bodies. The mismatch of locating features should be zero, if exactly constraining. When tight tolerances are used, the mismatches of over-constraining joints can also be trivial. In this case, good engineering judgments can be used to avoid costly FEA (i.e. analysis is done). In this step, the initial location $\left(u_{0}\right)$ of a point of interest should be measured as well.

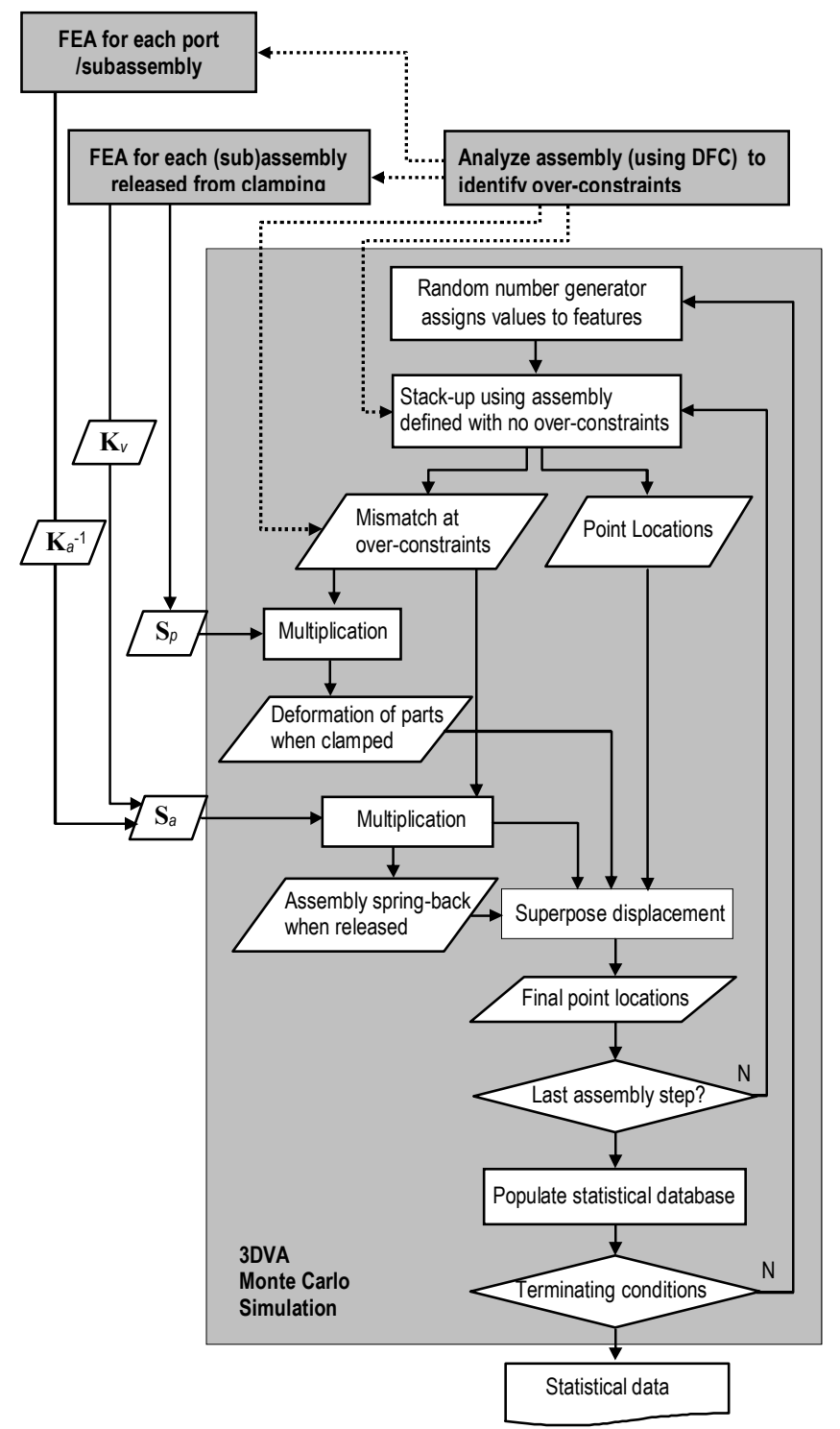

Fig. 2. Flow chart of the method proposed for variation analysis of non-rigid parts integrating DFC, commercial 3DVA and FEA.

\section{Upfront FEA for each part or subassembly pulled-up}

Thirdly, clamping forces $\left(f_{p}\right)$ (including reaction forces exerted on any supporting fixture to be removed) and deformation $\left(d_{p}\right)$ are measured. The clamping deformation should be measured because clamping at joints does not always bring a measurement point into its nominal position. In accordance with previous step, if one part is much more 
flexible than the other, it would be proper to measure only clamping force and deformation of flexible part, as the flexible part will be pulled-up to the rigid part. Measuring clamping force and deformation requires FEA. A popular approach is to run upfront FEA for each part with nominal geometry, to obtain linear relationship, i.e. stiffness matrices $\left(\mathbf{K}_{v}\right)$ for $v$ to $f_{p}$ and sensitivity matrices $\left(\mathbf{S}_{p}\right)$ for $v$ and $d_{p}$.

Strictly speaking, these matrices should vary according to geometrical variations (especially those of cross sections), but the variation in $f_{p}$ and $d_{p}$ due to variation in these matrices are typically very small compared to one caused by $v$. If this is not the case, the constant linear relationship should not be used. Otherwise, the method of influence coefficient [2] is typically used to obtain these matrices using parts' nominal geometry. To obtain the sensitivity matrices, a unit displacement is applied at each over-constrained DOF at each part. The forces at these locations are recorded; these are the forces needed to generate such displacement. These forces are then sorted in a matrix form to represent the stiffness matrix that can be used to obtain the desired clamping force. In addition, the deformation at the desired measuring points is recorded; this is the deformation response of the part to a unit displacement. The matrix generated from sorting these displacement vectors represents the part sensitivity matrix due to clamping. It can be used to obtain the part deformed response at the desired measuring points due to the deformation at the clamping points. Fig. 3 shows a simplified explanation of the method.

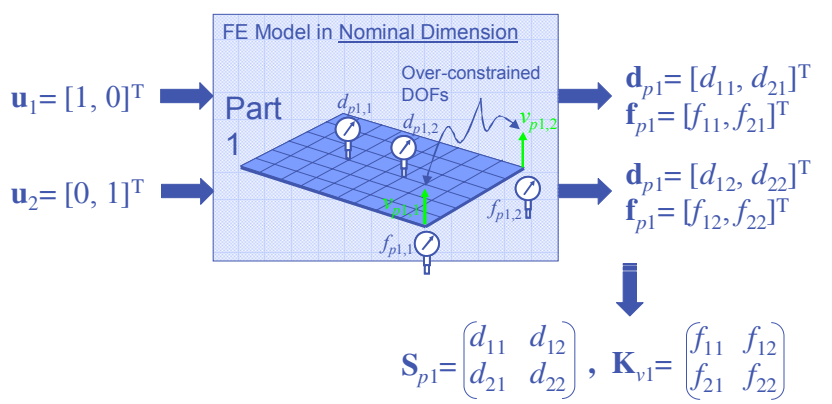

Fig. 3. Obtaining part stiffness matrix and part sensitivity matrix

\section{Upfront FEA for (sub)assembly released}

The forth step is to measure spring-back deformation $\left(d_{a}\right)$ corresponding to the clamping forces measured in previous step. Similarly to the previous, upfront FEA should be conducted with assembly's nominal geometry to obtain the compliance matrix of the assembly $\left(\mathbf{K}_{a}^{-1}\right)$. Again, the method of influence coefficient is typically used to obtain the matrix using assembly's nominal geometry. A unit force is applied at each constrained DOF in whole assembly to obtain the deformation response of the whole assembly to a unit force at any of the constrained DOF points. These vectors are sorted in a matrix form get the compliance matrix of the whole assembly. Multiplying the compliance matrix by the stiffness matrices obtained earlier results in the sensitivity matrix due to spring-back as shown in Fig. 4.

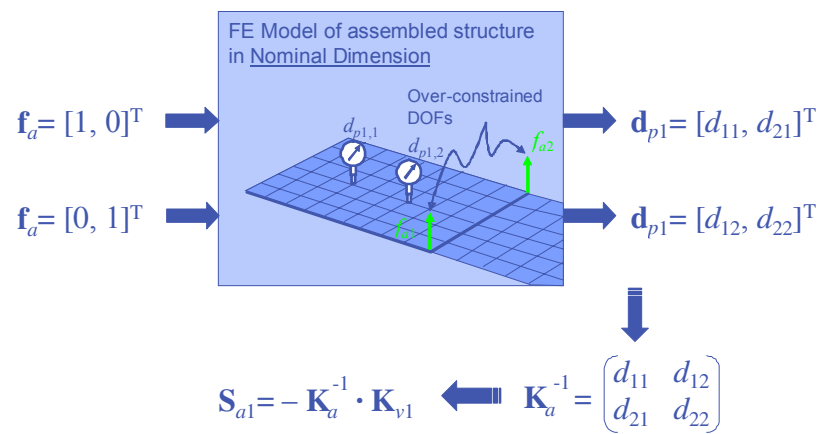

Fig. 4. Obtaining whole assembly sensitivity matrix

\section{E. Monte Carlo Simulation using 3DVA and sensitivity matrices from upfront FEA}

The summation of $u_{0}, d_{p}$ and $d_{a}$ results in the final location of the point $(u)$. Equations (1)-(5) govern the variation analysis of the assembly shown in Fig. $1 . \mathbf{f}_{p i}$ is the clamping forces of part $i, \mathbf{K}_{v i}$ is the stiffness matrix of part $i, \mathbf{v}_{i}$ is the joint mismatch of part $i, \mathbf{f}_{a}$ is the spring-back force, $\mathbf{K}_{a}$ is the assembly's stiffness, and $\mathbf{S}_{p i}$ and $\mathbf{S}_{a i}$ are the sensitivity matrix of the part $i$ during clamping and the sensitivity matrix of the assembly during spring-back, respectively.

$\mathbf{f}_{p i}=\mathbf{K}_{v i} \mathbf{v}_{i} \quad i=1,2$ for each over constraining DOF

$\mathbf{d}_{p i}=\mathbf{K}_{p i}^{-1} \mathbf{f}_{p i}=\mathbf{K}_{p i}^{-1} \mathbf{K}_{v i} \mathbf{v}_{i}=\mathbf{S}_{p i} \mathbf{v}_{i}$

$\mathbf{f}_{a}=-\sum \mathbf{f}_{p i}$

$\mathbf{d}_{a}=\mathbf{K}_{a}^{-1} \mathbf{f}_{a}=-\mathbf{K}_{a}^{-1} \sum_{i} \mathbf{K}_{v i} \mathbf{v}_{i}=-\sum_{i} \mathbf{S}_{a i} \mathbf{v}_{i}$

$\mathbf{u}=\mathbf{u}_{0}+\mathbf{d}_{\mathrm{p}}+\mathbf{d}_{\mathrm{a}}=\mathbf{u}_{0}+\sum\left(\mathbf{S}_{\mathrm{pi}}-\mathbf{S}_{\mathrm{ai}}\right) \mathbf{v}_{\mathrm{i}}$

In case of the completed assembly being much stiffer than its parts, the assembly can be considered as rigid. As a result, when released from the fixture, the assembly would not spring-back much. Thus, it may be unnecessary to estimate spring-back deformation, and step 2 is sufficient. This is true for well-designed structural enclosures, which is shown in the case study. However, in some cases, even if the assembly is very stiff compared to its parts (i.e., very small $\mathbf{S}_{a}$ ), large variations in joint mismatch, $\mathbf{v}$, may make spring-back deformation significant.

In equation (5), the variation of $\mathbf{u}$ can be estimated by root-sum-squaring the variations of $\mathbf{u}_{0}$ and $\mathbf{v}_{i}$, if $\mathbf{u}_{0}, \mathbf{v}_{i}$ and their elements are independent to each other. However, usually, they share many contributors such as variations in location. For example, in Fig. 1., if there is variation in location of part 3 , it would affect both $\mathbf{u}_{0}$ and $\mathbf{v}_{1}$, making them related to each other. For this reason, Monte-Carlo simulations are needed to obtain these variations.

\section{F. Computational Cost}

Because FEA are conducted prior to Monte Carlo simulations, additional computation over rigid-body 3DVA in Step $\mathrm{E}$ is only matrix multiplications and insignificant. However, depending on a model's size and the number of over-constrained joints, upfront FEA in Step C and D can be 
significant. In Step C, for each part, FEA should be conducted for every joint pulled-up. And, in Step D, FEA should be conducted for the assembly's every joint where at least one part is pulled-up.

\section{CAse Study}

In this case study, a generic wing-box assembly of commercial airplane is analyzed (Fig. 5), using the detailed procedure given in this paper, to determine the effect of part variation and flexibility on the assembly's variation. The assembly consists of a front and a rear spar, 33 ribs, and an upper and a lower skin subassembly, each of which has 17 stringers attached to a skin panel. Details are described in the following subsections corresponding those of section III.



Fig. 5. A generic wing box assembly for a commercial airplane

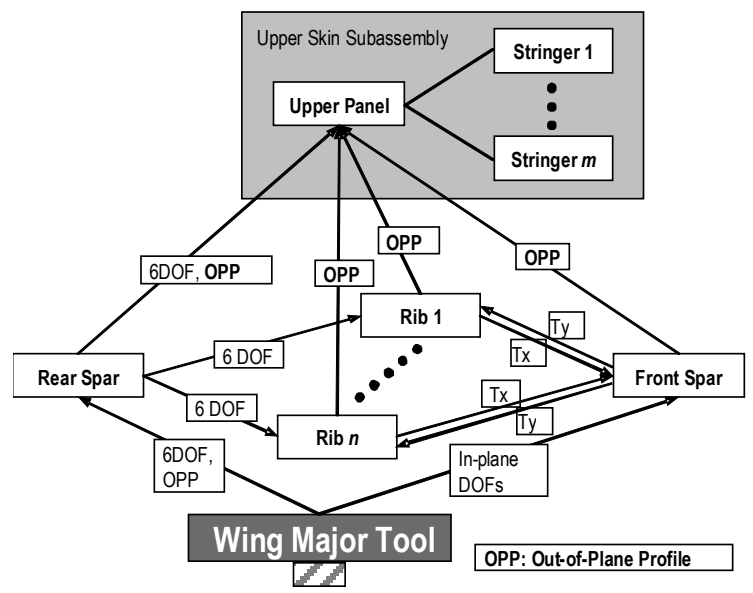

Fig. 6. Datum Flow Chain of the wing-box assembly. $\mathrm{T}_{\mathrm{x}}$ and $\mathrm{T}_{\mathrm{y}}$ stand for translational DOF in $\mathrm{X}$ and $\mathrm{Y}$ axis, respectively. Lower skin subassembly is omitted as it is located in the same way as the upper skin.

\section{A. Analyzing assembly to identify over-constraints}

Fig. 6 shows the DFC of the wing-box assembly. The assembly sequence used is as follows:

1. Locate the rear spar on the wing major tool fixture, constraining all 6 DOFs of rear spar.

2. Locate the front spar on the wing major tool, constraining in-plane DOFs of front spar.

3. Locate ribs on rear spar's rib posts, constraining all 6 DOF of each rib. Pull-up front spar's rib posts to ribs' the other ends, over-constraining out-of-plane DOFs of front spar and ribs.

4. Place upper and lower skin subassemblies on spar-ribs ladder structure. For in-plane DOFs, the subassemblies are located to a hole and a slot on rear spar. For out-of-plane DOFs, the subassemblies are pull-up to mating surfaces of spars and ribs. This will over-constrain the upper and lower skin subassemblies in their out-of-plane DOFs.

\section{B. Setting-up $3 D V A$}

Using a 3DVA (Teamcenter Visualization VSA [21] was used in this example), every part is located according to the assembly sequence without any over-constraints, and all the relevant part variations are specified (standard deviation equivalent to tolerance range divided by six was used in this example). Then, measurements are specified for the mismatch $(v)$ of each over-constraining joint identified in step 3 and 4.

- Ribs are located and assembled to the rear spar. And when the front spar is attached to the ribs' the other ends, ribs get over-constrained in their out-of-plane DOFs by front spar's rib-posts and the front spar get over-constrained in its out-of-plane DOF by ribs.

- The upper skin subassembly is located to rear spar's locating features for in-plane DOFs. Then, it will be pulled up to both spars and 33 ribs, over-constraining out-of-plane DOFs. After locating the sub-assembly using 3 corner points (Fig. 7) to constrain out-of-plane DOFs, every mismatch from upper skin to each of spars and ribs are measured in Z-axis. In this example, mismatches are measured at point $1-5$ (Fig. 7) for upper panel and each spar, and 1 point for upper skin and each rib, totaling $38 v$ 's for upper skin sub-assembly. This is the same for the lower one.

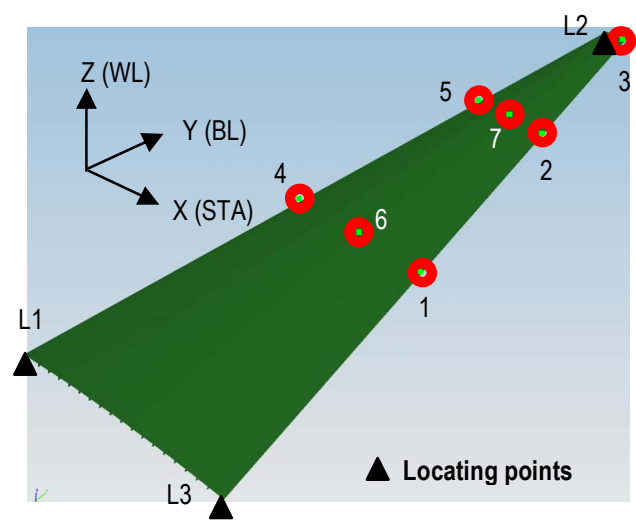

Fig. 7. Measuring points for upper skin profile.

Also to be measured in this step is the initial location $\left(u_{0}\right)$ of a point of interest. The measurements of interest in this case study are the skin profile, wing box twist, inbound box opening, and hinge-line straightness. For the skin profile, 10 points that span the whole skin are selected (Fig. 7). The wing box twist is calculated by measure the angle between a plane on the first rib and another, nominally parallel, plane on the last rib. The variation in the inbound box opening is measured by measuring variation at eight point that span the inbound profile. Hinge-line straightness is measured indirectly, by 
measuring points on the rear spar where hinges are attached.

\section{Upfront FEA for each part or subassembly pulled-up}

Upfront FEA (ANSYS Workbench [22] used for this case study) is conducted for each part and subassembly with nominal geometry, to obtain linear relationship, i.e. stiffness matrices $\left(\mathbf{K}_{v}\right)$ for $v$ to $f_{p}$ and sensitivity matrices $\left(\mathbf{S}_{p}\right)$ for $v$ and $d_{p}$ using the method of influence coefficients described in section III. For each over-constraining joint, unit displacement is applied to measure pull-up force for the joint as well as forces keep the displacement zero at the other over-constraining joints and locators. As an example, Fig. 8 shows FEA conducted to measure force to pull up upper skin sub-assembly to rib 10 , by .100", while keeping displacement at other joints zero. Note the same locating scheme is used as in 3DVA used in Fig. 7.

For each of upper and lower skin subassembly, there are 38 pull-up points (in Z-axis), so the $\mathbf{K}_{v \mathrm{US}}$ and $\mathbf{K}_{v \mathrm{LS}}$ is of $41 \times 38$, of which the last 3 rows are for reaction forces exerted on 3 imaginary locating points. Reaction forces in other axes are ignored as trivial. For rear spar - ribs subassembly, there are 33 pull-up forces (in $\mathrm{Y}$-axis), thus the $\mathbf{K}_{v \mathrm{RS}}$ is of $33 \times 33$. No reaction forces are measured, as the wing major tool that is assumed to support the entire assembly after spring-back supports this subassembly during clamping. For the front spar, pull-up forces are measured at 31 points (to 33 ribs less rib1 and rib2 that are used to locate the front spar), thus $\mathbf{K}_{v \mathrm{FS}}$ is of $34 \times 31$, of which the last three rows are for 3 locating points. While measuring pull-up forces to obtain $\mathbf{K}_{v}$ matrices, the displacement $d_{p}$ of measurement points that are not pull-up points should be also measured to obtain $\mathbf{S}_{p}$ for each part.

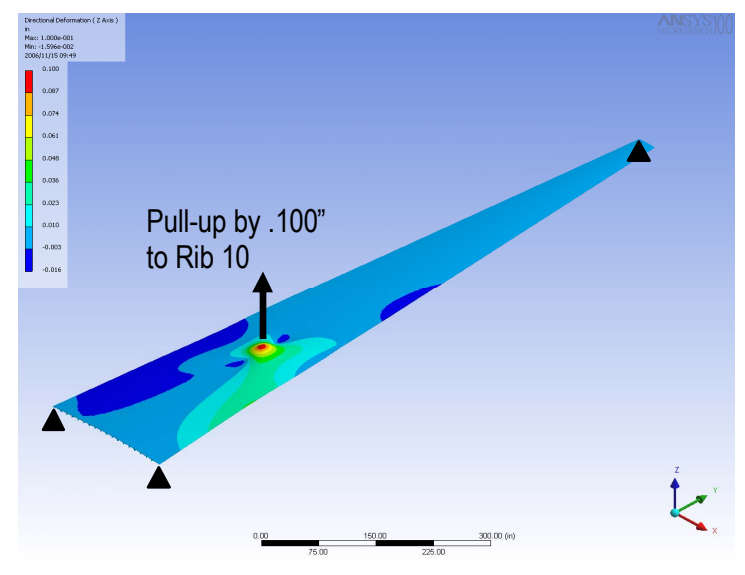

Fig. 8. Upper skin panel is pulled up to rib 10 by $.100 "$.

\section{Upfront FEA for (sub)assembly released}

As final step, upfront FEA is conducted for entire assembly with nominal geometry, to measure spring-back deformation $d_{a}$ corresponding to every clamping force $f_{p}$. Using the method of influence coefficient, compliance matrix $\left(\mathbf{K}_{a}{ }^{-1}\right)$ for $f_{p}$ to $d_{a}$, is obtained. In this case study, there are 149 pull up forces and 53 measurement points on the entire assembly.

\section{E. Monte Carlo Simulation using 3DVA and sensitivity matrices from upfront $F E A$}

Combining results from step $C$ and $D$, the sensitivity matrix can be calculated as follows;

$$
\begin{aligned}
\mathbf{S}_{a(53 \times 149)}= & {\left[\mathbf{C}_{a \mathrm{FS}(53 \times 34)} K_{v \mathrm{FS}(34 \times 3))}, \mathbf{C}_{a \mathrm{RS}(53 \times 33)} \mathbf{K}_{v \mathrm{RS}(33 \times 33)},\right.} \\
& \left.\mathbf{C}_{a \mathrm{US}(53 \times 41)} \mathbf{K}_{v \mathrm{US}(41 \times 38)}, \mathbf{C}_{a \mathrm{LS}(53 \times 41)} \mathbf{K}_{v \mathrm{LS}(41 \times 38)}\right]
\end{aligned}
$$

Once $\mathbf{S}_{a}$ is obtained, it is plugged back to 3DVA along with $\mathbf{S}_{p}$ obtained in Step $C$. Then, 3DVA runs Monte Carlo simulation that measures $v$ and $u_{0}$ first, and add $d_{p}$ and $d_{a}$ that are computed by matrix multiplication. For specific FEM codes, some 3DVA provide an automatic link. When it is not available, one can manually input the sensitivities using custom measurement features commonly available in 3DVA.

\section{F. Results}

By looking at the wing box structure, one can expect it would be much stiffer than skin subassemblies and spars especially in their out-of-plane DOFs. Although part or subassembly element is flexible, the way they are assembled makes one part's flexibility is compensated by another part. Not surprisingly, the sensitivities obtained in step $D$ were very small, with all the elements in the order of 0.01 or lower. To illustrate how small it is, spring-back deformation corresponding pull-up displacement of Fig. 10 is depicted in Fig. 11. For the pull-up deformation of 0.100 ", spring-back deformation is only -0.003 ".

When a part is pulled up to another part or a fixture closing the mismatch, the original variation is changed to that of the point to which the part is pulled up. For example, the point 1 on upper skin sub-assembly had .0367" of standard deviation before it's pulled up, and the primary contributor was skin's profile (see Table 1). When it's pulled up to a corresponding point on the front spar, which has much smaller variation, point 1's variation is reduced to that of front spar, 0.0082", and it inherits contributors of the corresponding point on the front spar (Table 2). The first contributor is the surface profile tolerance of the front spar's mating surface to the upper skin panel and the second one is the position tolerance specified on the front spar's secondary locating feature that determines the front spar's location in rotational DOF around X-axis. . When released from clamps, the wing-box assembly is found to be too strong that the spring-back is insignificant, making the point 1 almost stay where it's clamped. As a result, the amount and contributors of the variation after spring-back are almost identical to those of after clamping. Fig. 10 shows standard deviation of 10 points from Fig. 7, before and after clamping, and after spring-back. All other measurements mentioned in this case study exhibited similar behavior, making the wing box assembly very robust caused by pull-up forces.Monte Carlo simulation was conducted for 10000 iterations which took 497 seconds, on a Windows PC with a 3.2GHz Xeon CPU. For comparison, it took 448 seconds for rigid-body 3DVA of the same assembly. 


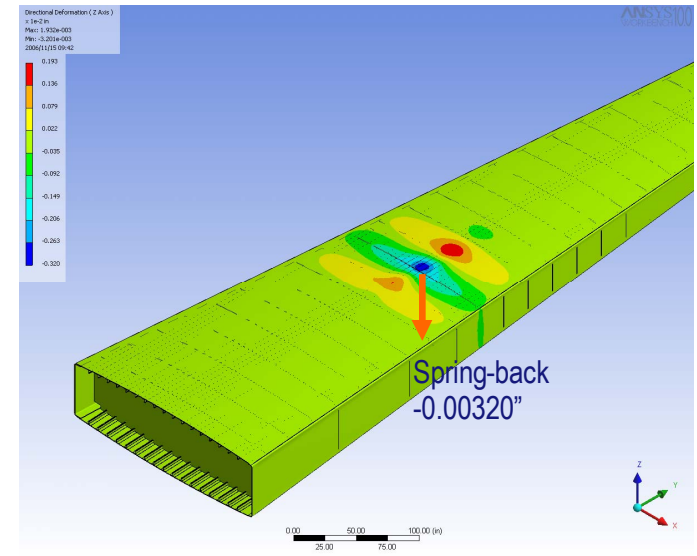

Fig. 9. Spring back deformation corresponding pull-up force applied in Fig. 8. As the entire assembly is much stiffer than the upper skin, ratio of spring-back deformation to pull-up deformation is very small.

Table 1. Major contributors to location of point 7 from Fig. 7, before clamping.

\begin{tabular}{|l|r|}
\hline Contributors & Effect \\
\hline Upper skin: outer surface & $91.29 \%$ \\
\hline Rear spar: secondary locating feature & $5.99 \%$ \\
\hline Rear spar: outer surface & $2.50 \%$ \\
\hline
\end{tabular}

Table 2. Major contributors to upper skin's profile at point 1, after clamping.

\begin{tabular}{|l|r|}
\hline Contributors & Effect \\
\hline Front Spar: outer surface & $73.01 \%$ \\
\hline Front Spar: secondary locating feature & $25.39 \%$ \\
\hline Rib 1: secondary locating feature & $1.26 \%$ \\
\hline
\end{tabular}

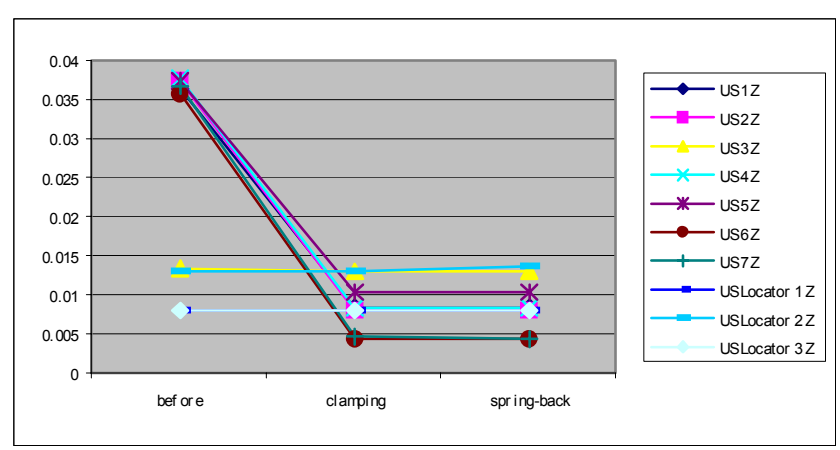

Fig. 10. Standard deviation of upper skin's 10 points in Z-axis, before, after clamping and spring-back.

\section{CONCLUSION}

This paper presented a holistic process map to integrate assembly design analysis, commercial 3DVA and FEA tools. The method was applied to a commercial airplane's wing-box assembly, and the effect of part variation and flexibility on the assembly's variation was determined. In the way the wing box assembly designed, slender parts are arranged to each other perpendicularly, negating out-of-plane flexibility each other. For example, out-of-plane flexibility of skin panels is compensated by in-plane rigidity of ribs. It makes the wing box robust not only to external loads, but also to pull-up forces applied during assembly operations. This knowledge may be used to predict variations of other structural enclosures made of flexible parts.

\section{REFERENCES}

[1] S. C. Liu, S. J. Hu, and T. C. Woo, "Tolerance analysis for sheet metal assemblies," ASME Journal of Mechanical Design, vol. 118, pp. 62-67, 1996.

[2] S. C. Liu and S. J. Hu, "Variation simulation for deformable sheet metal assemblies using finite element methods," ASME Journal of Manufacturing Science and Engineering, vol. 119, pp. 368-374, 1997.

[3] Y. J. Long and S. J. Hu, "A unified model for variation simulation of sheet metal assemblies", in Geometric Design Tolerancing: Theories, Standards and Applications, H. A. ElMaraghy, Ed. Chapman \& Hall, 1998, pp.208-219.

[4] K. G. Merkely, K. W. Chase, and E. Perry, "An introduction to tolerance analysis of flexible assemblies," Proceedings of the 1996 MSC World Users Conferences.

[5] M. Chang and D. C. Gossard, "Modeling the assembly of compliant, non-ideal parts," Computer-Aided Design, vol. 29, no. 10, pp. 701-708, 1997.

[6] J. A. Camelio, S. J. Hu, and S. P. Marin, "Compliant assembly variation analysis using component geometric covariance," ASME Journal of Manufacturing Science and Engineering, vol. 126, no. 2, pp. 355-360, 2004.

[7] W. Huang and D. Ceglarek, "Model complexity reduction in stream-of-variation analysis for compliant sheet metal assembly," Proceedings of the $9^{\text {th }}$ CIRP International Seminar on Computer Aided Tolerancing, 2005.

[8] L. J. Kamm, Designing Cost-Effective Mechanisms, McGraw-Hill, 1990.

[9] J. M. Kriegel, "Exact constraint design," Mechanical Engineering, vol. 117, no. 5, pp. 88-90, 1995.

[10] D. L. Blanding, Exact Constraint: Machine Design Using Kinematic Principles, New York, ASME Press, 1999.

[11] J. G. Skakoon, Detailed Mechanical Design: A Practical Guide, New York, ASME Press, 2000.

[12] R. Mantripragada and D. E. Whitney, "The datum flow chain," Research in Engineering Design, vol. 10, pp. 150-165, 1998.

[13] D. E. Whitney, R. Mantripragada, J. D. Adams, and S. J. Rhee, "Designing assemblies," Research in Engineering Design, vol. 11, pp. 229-253, 1999.

[14] D. E. Whitney, Mechanical Assemblies: Their Design, Manufacture, and Role in Product Development, New York, Oxford University Press, 2004.

[15] R. Mantripragada and D. E. Whitney, "Modeling and controlling variation propagation in mechanical assemblies using state transition models," IEEE Transactions on Robotics and Automation, vol. 15, no. 1, pp. 124-140, 1999.

[16] J. Jin and J. Shi, "State space modeling of sheet metal assembly for dimensional control," ASME Journal of Manufacturing Science and Engineering, vol. 121, no. 4, pp. 756-762, 1999.

[17] Y. Ding, D. Ceglarek, and J. Shi, "Modeling and diagnosis of multistage manufacturing processes: part I: state space model," in Proceedings of the Japan/USA Symposium on Flexible Automation, Ann Arbor, MI, 2000.

[18] J. Camelio, S. J. Hu, and D. Ceglarek, "Modeling Variation Propagation of Multistation Assembly Systems with Compliant Parts," Transactions of ASME, Journal of Mechanical Design, vol. 125, No. 4, pp. 673-681, 2003.

[19] B. Lee and K. Saitou, "Three-dimensional assembly synthesis for robust dimensional integrity based on Screw Theory," ASME Journal of Mechanical Design, vol. 128, no. 1, pp. 243-251, 2006.

[20] B. Lee and K. Saitou, "Integrated synthesis of assembly and fixture scheme for properly constrained assembly," IEEE Transactions on Automation Science and Engineering, vol. 2, no. 3, pp. 250-261, 2005.

[21] http://www.ugs.com/products/teamcenter/sol_prod/visualization/vsa/

[22] http://www.ansys.com/products/workbench.asp/ 